\title{
Panorama epidemiológico de las mordeduras por serpientes en la península de Baja California, México (2003-2018)
}

\author{
Jesús M. Rodríguez-Canseco', Gustavo Arnaud-Franco², Everardo Gutiérrez-López¹ y \\ Guillermo Romero-Figueroa ${ }^{1 *}$ \\ ${ }^{1}$ Universidad Autónoma de Baja California, Facultad de Ciencias, Ensenada, Baja California; ${ }^{2}$ Centro de Investigaciones Biológicas del Noroeste, \\ Programa Planeación Ambiental, La Paz, Baja California Sur. México
}

\section{Resumen}

Introducción: El accidente ofídico es un problema de salud pública mundial. En México existe una alta incidencia de mordeduras por serpientes, que provocan complicaciones médicas que pueden dejar secuelas severas. Objetivo: Analizar el panorama epidemiológico del ofidismo en la península de Baja California (BC) en los años 2003-2018. Método: Se hizo un análisis de tipo descriptivo y retrospectivo de los casos presentados, a partir de la recopilación e interpretación de la información. Resultados: Se obtuvieron 541 registros, de los cuales 273 se presentaron en BC y 268 en Baja California Sur (BCS), con un promedio anual de 17.06 y 16.75 respectivamente. La tasa de incidencia para BC fue de 7.62 y para BCS de 33.09. La tasa de incidencia anual más alta en el Estado de BC correspondió al sur de Ensenada, con un valor de 42.3, mientras que en BCS fue en Comondú, con un valor de 54.04. Conclusiones: Los análisis epidemiológicos permiten tener un mejor entendimiento local, estatal y regional de la problemática, para poder desarrollar protocolos de acción eficientes para enfrentar un accidente ofídico, así como para determinar las necesidades de capacitación del personal médico y establecer una red de ayuda para el tratamiento de casos.

PALABRAS CLAVE: Accidente ofídico. Serpientes venenosas. Tasa de incidencia. Crotalus.

\section{Epidemiological overview of snakebites in the Baja California peninsula, Mexico (2003-2018)}

\begin{abstract}
Introduction: Ophidian accident is a global public health problem. In Mexico, there is a high incidence of snakebites, which cause medical complications that can leave severe sequelae. Objective: To analyze the epidemiological overview of snake venom poisoning in the Baja California (BC) peninsula within the 2003-2018 period. Method: $A$ descriptive, retrospective analysis of reported cases was carried out, based on data collection and interpretation. Results: $A$ total of 541 records were obtained, out of which 273 occurred in BC and 268 in Baja California Sur (BCS), with an annual average of 17.06 and 16.75, respectively. The incidence rate for $B C$ was 7.62, while for $B C S$ it was 33.09. The highest annual incidence rate in the state of $B C$ corresponded to southern Ensenada, with a value of 42.3 , while in BCS it corresponded to the municipality of Comondú, with a value of 54.04. Conclusions: Epidemiological analyses allow a better local, state and regional understanding of the problem, in order to develop efficient action protocols to face an ophidian accident, as well as to determine the training requirements of medical personnel and establish a support network for the treatment of cases.
\end{abstract}

KEY WORDS: Ophidian accident. Poisonous snakes. Incidence rate. Crotalus.

Correspondencia:

*Guillermo Romero-Figueroa

E-mail: gromero4@uabc.edu.mx
Gac Med Mex. 2021;157:579-585

Disponible en PubMed

www.gacetamedicademexico.com CC BY-NC-ND (http://creativecommons.org/licenses/by-nc-nd/4.0/). 


\section{Introducción}

Los accidentes ofídicos corresponden a una lesión causada por la mordedura de una serpiente (venenosa o no venenosa), originando algún tipo de afectación. En el caso de las serpientes venenosas, la mordida incluye la inoculación de una sustancia tóxica o veneno, que produce daño a los tejidos y ocasiona alteraciones fisiopatológicas locales o sistémicas de gravedad variable1. La Organización Mundial de la Salud considera este tipo de accidente un problema de salud pública que ocasiona entre 1.8 y 2.7 millones de envenenamientos anuales en el mundo, originando entre 80 y 140 mil muertes cada año². Las regiones más afectadas son Asia, África y Latinoamérica, donde se encuentra la mayor diversidad de estos reptiles, siendo los habitantes rurales el principal grupo vulnerable ${ }^{3}$. En México la ocurrencia de ofidismo ha variado a lo largo del tiempo. En el periodo 1994-95 se registraron 627 casos, con 30 defunciones, en cambio en 1997 se reportó un incremento considerable de casos, con 27,480 registros y 136 defunciones ${ }^{4}$, mientras que el promedio de registro anual en los años comprendidos entre 2003 y 2018 fue de $3,700^{5}$.

México tiene el primer lugar del continente americano en herpetofauna venenosa, representada por dos familias de serpientes con veneno potencialmente mortal para el humano: a) Viperidae (representada por 10 géneros y 64 especies), que incluye a las víboras de cascabel, nauyacas y cantiles, y b) Elapidae (representada por tres géneros y 10 especies), que incluye a los coralillos o serpientes de coral $^{6}$. Los Estados donde se registra la mayor incidencia de mordidas de serpientes son Veracruz, Oaxaca, San Luis Potosí, Hidalgo y Puebla ${ }^{7}$, atribuible a sus características geográficas y climáticas, que favorecen una amplia diversidad de ofidios ${ }^{8}$. En cambio, en la península de Baja California (BC), donde se distribuyen 20 géneros y 28 especies de serpientes ${ }^{9}$, solo hay siete especies venenosas que corresponden a serpientes de cascabel ${ }^{10}$. En esta región el registro de mordeduras es menor que en otros Estados de México ${ }^{4}$.

La península de $\mathrm{BC}$ es un territorio con una extensa superficie $\left(200,000 \mathrm{~km}^{2}\right)$, constituido por tres regiones principales de acuerdo con su clima y composición biológica: 1) región mediterránea-templada, ubicada en el noroeste, con clima templado subhúmedo y con presencia de lluvias en invierno; 2) región tropical, localizada en el extremo sur en la zona conocida como región del Cabo, con lluvias de invierno y verano, y 3) región desértica, localizada entre las dos anteriores, con clima muy seco-semicálido, con Iluvias de invierno y verano, abarcando el $60 \%$ de la superficie de la península ${ }^{11-13}$. Estas tres regiones se subdividen en 14 eco-regiones, definidas por la composición de sus especies y su dinámica ecológica ${ }^{14}$.

A pesar de que el registro de mordeduras es menor en la península en comparación con otras regiones de México ${ }^{4}$, las complicaciones médicas derivadas de estos accidentes pueden ser severas, no solo por la posibilidad de muerte, sino por las secuelas que puede originar el envenenamiento ${ }^{15}$, así como por las ocasionadas debido a la aplicación de una atención médica inadecuada ${ }^{16}$. En relación con las dos entidades federativas peninsulares, $B C$ tiene una población de 3,583,605 habitantes (46 personas por $\mathrm{km}^{2}$ ) concentrada en un $92.2 \%$ en zonas urbanas y un $7.7 \%$ en zonas rurales, mientras que en Baja California Sur (BCS) la población es de 809,833 habitantes (10 personas por $\mathrm{km}^{2}$ ), concentrada el $86.1 \%$ en zonas urbanas y el $13.8 \%$ en zonas rurales ${ }^{17-19}$. Aquí la falta de disponibilidad de antivenenos o faboterápicos, así como las grandes distancias entre los centros urbanos en los que se puede acceder a una atención hospitalaria, agudizan el problema, ya que los hospitales que pueden brindar la mejor atención médica se encuentran en el extremo norte (Tijuana, Ensenada y Mexicali) y en el extremo sur (La Paz y Los Cabos), separados por más de $1,000 \mathrm{~km}$ de distancia.

En este contexto, el objetivo del presente estudio fue el de analizar el panorama epidemiológico del ofidismo en la península de BC en los años 2003-2018.

\section{Materiales y métodos}

Los datos de incidencias de mordedura de serpiente en los estados de BC y BCS se obtuvieron del Boletín Epidemiológico, publicado por el Sistema Nacional de Vigilancia Epidemiológica y el Sistema Único de Información de la Secretaría de Salud del Gobierno Federal de México. Dado que la información de dicho boletín no incluye la localización geográfica por municipio o jurisdicción donde ocurrió la afectación, se complementaron los datos con información del Departamento de Epidemiología Estatal del Instituto de Servicios de Salud Pública del Estado de BC y de la Dirección de Servicios de Salud en BCS, para así agruparlos por Estado, municipio y jurisdicción. BC se integra por cinco municipios, divididos en 
Tabla 1. Total de accidentes ofídicos y tasa anual en Baja California (BC), Baja California Sur (BCS) y México (Mx)

\begin{tabular}{|c|c|c|c|c|c|c|}
\hline Año & Total BC & Tasa BC & Total BCS & Tasa BCS & Total Mx & Tasa Mx \\
\hline 2003 & 17 & 0.47 & 26 & 3.21 & 2,666 & 2.22 \\
\hline 2004 & 9 & 0.25 & 26 & 3.21 & 3,229 & 2.69 \\
\hline 2005 & 19 & 0.53 & 16 & 1.98 & 3,515 & 2.93 \\
\hline 2006 & 10 & 0.28 & 21 & 2.59 & 3,701 & 3.09 \\
\hline 2007 & 7 & 0.2 & 19 & 2.35 & 3,351 & 2.79 \\
\hline 2008 & 26 & 0.73 & 15 & 1.85 & 3,797 & 3.17 \\
\hline 2009 & 11 & 0.31 & 14 & 1.73 & 3,725 & 3.11 \\
\hline 2010 & 15 & 0.42 & 18 & 2.22 & 3,449 & 2.88 \\
\hline 2011 & 24 & 0.67 & 7 & 0.86 & 3,535 & 2.95 \\
\hline 2012 & 24 & 0.67 & 7 & 0.86 & 4,225 & 3.52 \\
\hline 2013 & 25 & 0.7 & 15 & 1.85 & 3,961 & 3.3 \\
\hline 2014 & 12 & 0.33 & 14 & 1.73 & 4,430 & 3.69 \\
\hline 2015 & 21 & 0.59 & 11 & 1.36 & 4,378 & 3.65 \\
\hline 2016 & 12 & 0.33 & 18 & 2.22 & 3,790 & 3.16 \\
\hline 2017 & 15 & 0.42 & 16 & 1.98 & 3,700 & 3.08 \\
\hline 2018 & 26 & 0.73 & 25 & 3.09 & 3,726 & 3.11 \\
\hline Total & 273 & 7.62 & 268 & 33.09 & 59,178 & 49.34 \\
\hline Promedio & 17.06 & 0.48 & 16.75 & 2.07 & $3,698.63$ & 3.08 \\
\hline Población & $3,584,605$ & & 809,833 & & $119,938,473$ & \\
\hline
\end{tabular}

cuatro jurisdicciones, mientras que BCS está integrado también por cinco municipios que representan cada uno una jurisdicción.

Se estimaron los promedios anuales de los registros de mordeduras, así como la tasa de incidencia (número total de casos dividido entre el total de la población y multiplicado por cada 100,000 habitantes), para así evitar el sesgo en la diferencia en el número de habitantes, de la península, Estado y jurisdicción. Se comparó el total de registros con los de todo el país en el periodo 2003-20018. La distribución anual de la tasa de incidencia, así como de los promedios semanales, se expresaron en un gráfico de líneas.

La distribución geográfica de los accidentes ofídicos fue incorporada a un mapa utilizando ArcGIS $^{\circledR}$ $10.2\left(\mathrm{ESRI}^{\odot}\right)$ y el programa SIGepi v. 1.4, para estimar las tasas suavizadas de incidencia, aplicando un suavizado local con la técnica de media móvil con una distancia en vecindad de $10 \mathrm{~km}$ (el suavizado tiene la finalidad de disminuir la variabilidad generada por el tamaño poblacional desigual entre municipios). Para estabilización, el cálculo de las tasas municipales se llevó a cabo con la acumulación de la información de los años estudiados ${ }^{20,21}$.

\section{Resultados}

Se obtuvo un total de 541 registros de accidentes ofídicos en el periodo 2003-2018 en la península de BC, de los cuales 273 ocurrieron en BC y 268 en BCS, con un promedio anual de 17.06 y 16.75 respectivamente, lo cual representó el 0.46 y $0.45 \%$ del total de los casos en México, con una tasa de incidencia para BC de 7.62 y para BCS de 33.09 (Tabla 1). Los años con los mayores registros en BC ocurrieron en 2008 y 2018 con 26 casos, mientras que para BCS fueron los años 2003, 2004 y 2018, con 26 y 25 registros. Los años con menor número de casos fueron en 2004 y 2007 para BC, y en 2011 y 2012 para BCS (Tabla 1).

Si bien la tasa anual de ofidismo fue mayor en BCS en relación con $\mathrm{BC}$, también lo fue en relación con la 


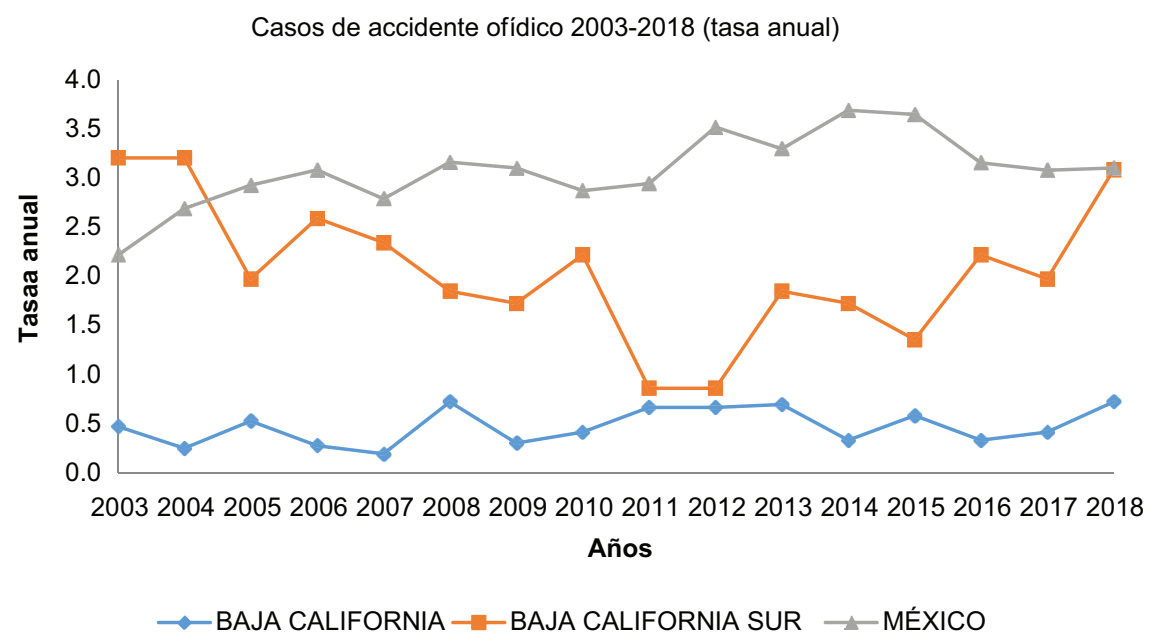

Figura 1. Tasa anual de accidentes ofídicos en Baja California, Baja California Sur y México.

tasa nacional para los años 2003 y 2004 e igual en 2018 (Fig. 1).

En relación con la temporalidad de los casos en las dos entidades, fueron constantes durante todos los meses del año, con incremento al final de la primavera (junio) y disminución en otoño (octubre) (Fig. 2). De estos registros, los hombres fueron los más afectados, con un total de 374 casos (69\%), mientras que $167(31 \%)$ fueron mujeres.

En relación con la tasa de incidencia anual acumulada por jurisdicción de BC, la más alta se presentó en la IV, que correspondió al sur del municipio de Ensenada, con un valor de 42.3, mientras que la menor tasa ocurrió en la jurisdicción II, correspondiente a los municipios de Tijuana, Tecate y Playas del Rosarito, con un valor de 3.71 (Tabla 2) (Fig. 3). En relación con BCS, los registros por municipio abarcaron solo los años 2003-2005 y 2012-2016, con un total de 132 casos. Las tasa más alta se presentó en el municipio de Comondú, con un valor de 54.04, mientras la menor tasa ocurrió en el municipio de Loreto, donde no hubo ningún caso (Tabla 3) (Fig. 3).

\section{Discusión}

El ofidismo es un problema de salud pública en México, debido al número de casos anuales ${ }^{4,5,16}$. Su impacto en la salud de las personas afectadas depende de varios factores, como la cantidad y características del veneno inoculado, que puede variar de acuerdo con la especie de que se trate ${ }^{22}$, así como de la rapidez en la atención y el acceso a un tratamiento adecuado ${ }^{23}$, lo cual en la península de BC depende de la localidad donde ocurra el accidente ofídico, ya que las distancias a los centros hospitalarios pueden ser muy grandes, lo que implica un amplio número de horas antes de poder recibir la atención médica.

La interacción entre las serpientes y humanos en esta región de México ocurre principalmente en el medio rural. En la península de BC la información epidemiológica sobre el envenenamiento ofídico es limitada, ya que no todos los afectados acuden a una instancia médica, debido a lo alejado de las localidades, por lo que no son registrados en el sistema de salud.

La ocurrencia mensual de casos en la península de $\mathrm{BC}$ es similar a otras regiones del país, con preponderancia durante los meses más cálidos y lluvio$\operatorname{sos}^{24-26}$, sin embargo, en la península la ocurrencia de mordeduras es durante todo el año. Los hombres son mayormente afectados que las mujeres, lo cual es similar a otras regiones de México ${ }^{1,24-26}$ y otros países $^{27-30}$.

Para el registro nacional de casos, el Boletín Semanal Epidemiológico, publicado por la Secretaría de Salud por medio del Sistema Nacional de Vigilancia Epidemiológica, es de gran utilidad, porque permite conocer el número de casos de mordedura de serpiente por entidad federativa, sin embargo hay información que no considera, pero que es recabada por los Servicios de Salud de los Estados, clasificándola a nivel de jurisdicción o municipio. Aun así existen vacíos importantes en la información recabada, tales como la especie involucrada, que podría permitir identificar que especies son las más involucradas. 
Tabla 2. Casos y tasa de incidencia anual de accidentes ofídicos en Baja California por jurisdicción

\begin{tabular}{|c|c|c|c|c|c|c|c|c|c|c|}
\hline Año & Mexicali & Tasa & $\begin{array}{l}\text { Tijuana, Tecate y } \\
\text { Playas de Rosarito }\end{array}$ & Tasa & $\begin{array}{l}\text { Norte de } \\
\text { Ensenada }\end{array}$ & Tasa & Sur de Ensenada & Tasa & Total & tasa \\
\hline 2003 & 2 & 0.19 & 5 & 0.25 & 5 & 1.17 & 2 & 1.84 & 14 & 0.4 \\
\hline 2004 & 3 & 0.28 & 0 & 0 & 4 & 0.94 & 3 & 2.76 & 10 & 0.3 \\
\hline 2005 & 5 & 0.47 & 2 & 0.1 & 12 & 2.81 & 4 & 3.68 & 23 & 0.6 \\
\hline 2006 & 3 & 0.28 & 0 & 0 & 7 & 1.64 & 1 & 0.92 & 11 & 0.3 \\
\hline 2007 & 2 & 0.19 & 2 & 0.1 & 2 & 0.47 & 2 & 1.84 & 8 & 0.2 \\
\hline 2008 & 12 & 1.14 & 9 & 0.45 & 3 & 0.7 & 0 & 0 & 24 & 0.7 \\
\hline 2009 & 8 & 0.76 & 2 & 0.1 & 1 & 0.23 & 1 & 0.92 & 12 & 0.3 \\
\hline 2010 & 7 & 0.66 & 3 & 0.15 & 5 & 1.17 & 2 & 1.84 & 17 & 0.5 \\
\hline 2011 & 4 & 0.38 & 14 & 0.7 & 4 & 0.94 & 4 & 3.68 & 26 & 0.7 \\
\hline 2012 & 8 & 0.76 & 3 & 0.15 & 5 & 1.17 & 8 & 7.36 & 24 & 0.7 \\
\hline 2013 & 7 & 0.66 & 4 & 0.2 & 10 & 2.34 & 4 & 3.68 & 25 & 0.7 \\
\hline 2014 & 4 & 0.38 & 6 & 0.3 & 2 & 0.47 & 1 & 0.92 & 13 & 0.4 \\
\hline 2015 & 7 & 0.66 & 5 & 0.25 & 6 & 1.41 & 6 & 5.52 & 24 & 0.7 \\
\hline 2016 & 4 & 0.38 & 7 & 0.35 & 1 & 0.23 & 1 & 0.92 & 13 & 0.4 \\
\hline 2017 & 6 & 0.57 & 7 & 0.35 & 2 & 0.47 & 0 & 0 & 15 & 0.4 \\
\hline 2018 & 8 & 0.76 & 5 & 0.25 & 6 & 1.41 & 7 & 6.44 & 26 & 0.7 \\
\hline Total & 90 & 8.55 & 74 & 3.71 & 75 & 17.6 & 46 & 42.3 & 285 & 8 \\
\hline
\end{tabular}

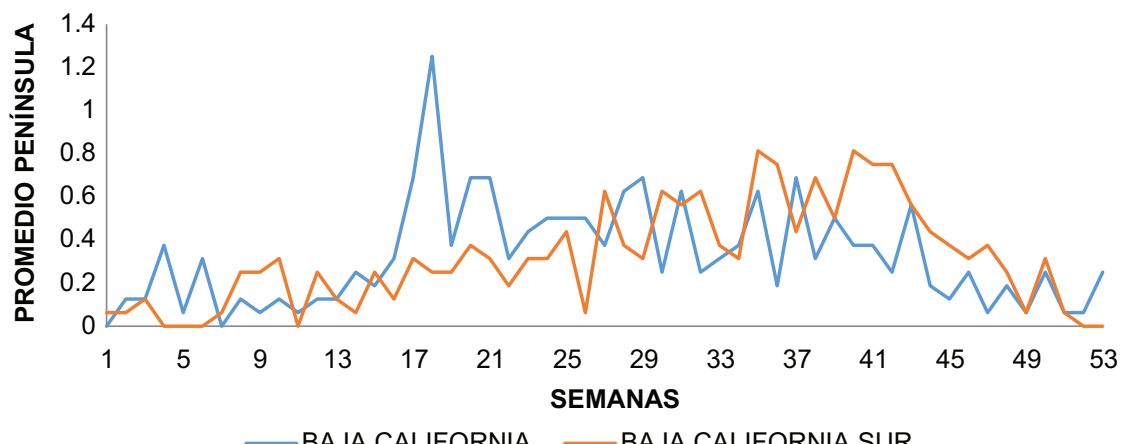

Figura 2. Promedio semanal de accidentes ofídicos en Baja California y Baja California Sur.

Fotografías de la serpiente involucrada pueden ser identificadas por especialistas de la región. También se requiere registrar, además del sexo de la persona, su edad, localización anatómica de la mordedura, actividad que se encontraba realizando el afectado al momento de la mordida, tiempo entre la mordida y la aplicación del antiveneno o faboterápico, número de dosis de antiveneno aplicadas y tiempo de permanencia en la unidad médica.
En este contexto, los análisis epidemiológicos permiten tener un mejor entendimiento de la problemática a nivel local, estatal y regional, para poder desarrollar protocolos de acción eficientes para enfrentar un accidente ofídico, así como para determinar las necesidades de capacitación del personal médico y establecer una red de ayuda para el tratamiento de casos, lo cual es particularmente importante en la península de $\mathrm{BC}$ debido a su extensión de 
Tabla 3. Casos de accidentes ofídicos y tasas de incidencia anuales por municipio en Baja California Sur

\begin{tabular}{|c|c|c|c|c|c|c|c|c|c|c|c|c|}
\hline Año & Comondú & Tasa & Mulegé & Tasa & La Paz & Tasa & Los Cabos & Tasa & Loreto & Tasa & Total & Tasa \\
\hline 2003 & 11 & 13.2 & 2 & 2.84 & 10 & 3.27 & 4 & 1.22 & 0 & 0 & 27 & 3.33 \\
\hline 2004 & 11 & 13.2 & 1 & 1.42 & 11 & 3.6 & 3 & 0.91 & 0 & 0 & 26 & 3.21 \\
\hline 2005 & 2 & 2.4 & 0 & 0 & 1 & 0.33 & 2 & 0.61 & 0 & 0 & 5 & 0.62 \\
\hline 2012 & 4 & 4.8 & 5 & 7.1 & 3 & 0.98 & 2 & 0.61 & 0 & 0 & 14 & 1.73 \\
\hline 2013 & 4 & 4.8 & 3 & 4.26 & 2 & 0.65 & 7 & 2.13 & 0 & 0 & 16 & 1.98 \\
\hline 2014 & 6 & 7.21 & 0 & 0 & 7 & 2.29 & 3 & 0.91 & 0 & 0 & 16 & 1.98 \\
\hline 2015 & 3 & 3.6 & 1 & 1.42 & 2 & 0.65 & 4 & 1.22 & 0 & 0 & 10 & 1.23 \\
\hline 2016 & 4 & 4.8 & 4 & 5.68 & 5 & 1.64 & 5 & 1.52 & 0 & 0 & 18 & 2.22 \\
\hline Total & 45 & 54 & 16 & 22.7 & 41 & 13.4 & 30 & 9.1 & 0 & 0 & 132 & 16.3 \\
\hline
\end{tabular}

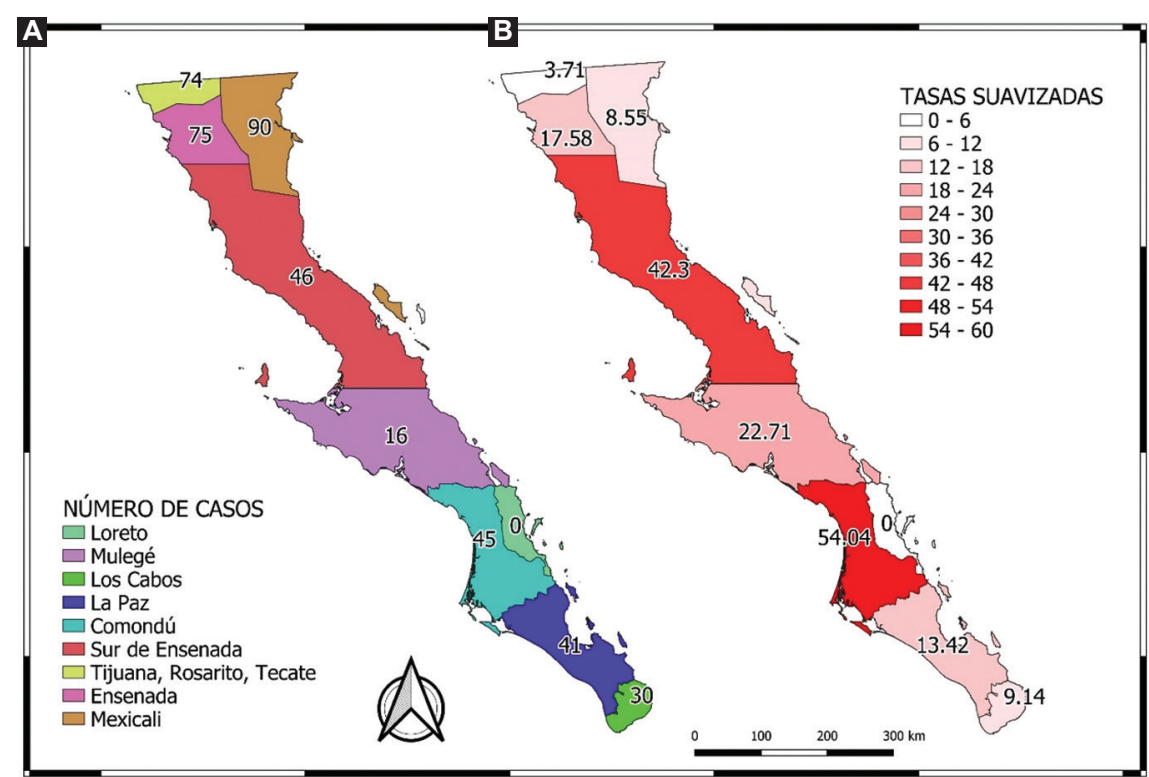

Figura 3. Variación geográfica del número de casos de mordeduras por serpiente (A) y tasa de incidencia (accidentes/100,000 habitantes) (B), durante el periodo 2003-2018 en la península de Baja California.

más de mil kilómetros. Si bien el principal tratamiento para enfrentar el accidente ofídico es la administración del antiveneno o faboterápico, ya que después de la mordedura las dos primeras horas son cruciales para atenuar los efectos del veneno ${ }^{23}$, aún hay médicos que están recelosos para su utilización.

Si bien en la península de BC el ofidismo es relativamente bajo, en BCS, el Estado con menor densidad poblacional del país, su tasa se acerca a la de la media nacional e incluso la rebasa en un año. En BC, a pesar de tener casi el mismo número de casos que $\mathrm{BCS}$, su tasa es menor debido a que su población es de 3:1 en relación con BCS.

\section{Conclusiones}

La frecuencia de los accidentes ofídicos en la península de $B C$ es menor que en otros Estados de México, sin embargo en BCS su tasa de incidencia es alta debido a que es el Estado con la menor densidad demográfica.

Existen vacíos de información de cada caso por mordedura de serpiente atendido en los servicios médicos, entre los que se incluye la especie de la serpiente involucrada.

La información epidemiológica relativa al accidente ofídico es esencial para evaluar la magnitud municipal y regional del problema, con el fin de elaborar 
directrices para la gestión de recursos y la capacitación del personal médico para el tratamiento de mordeduras de serpiente.

\section{Agradecimientos}

Los autores agradecen a CONACYT por el apoyo brindado mediante la beca estudiantil para el desarrollo de este proyecto. A la Universidad Autónoma de Baja California y a las Secretarías de Salud estatales por las facilidades brindadas.

\section{Financiamiento}

La presente investigación recibió financiamiento de CONACYT por medio de una beca estudiantil n. 483968.

\section{Conflicto de intereses}

Los autores declaran que no existe ningún conflicto de intereses relacionado con lo expuesto en este artículo.

\section{Responsabilidades éticas}

Protección de personas y animales. Los autores declaran que para esta investigación no se han realizado experimentos en seres humanos ni en animales.

Confidencialidad de los datos. Los autores declaran que han seguido los protocolos de su centro de trabajo sobre la publicación de datos de pacientes.

Derecho a la privacidad y consentimiento informado. Los autores declaran que en este artículo no aparecen datos de pacientes.

\section{Bibliografía}

1. Secretaría de Salud. Diagnóstico y tratamiento de las mordeduras de serpientes venenosas. Guía de Práctica Clínica. México: Secretaría de Salud, Consejo de Salubridad General; 2010.

2. Organización Mundial de la Salud. Mordedura de serpientes venenosas [Internet]. Organización Mundial de la Salud; 2018 Disponible en: https:// www.who.int/es/news-room/fact-sheets/detail/snakebite-envenoming

3. Chippaux JP. Estimating the global burden of snakebite can help to improve management. PloS Med. 2008;5(11):e221.

4. González-Rivera A, Chico-AldamaP, Domínguez-Viveros W, Iracheta-Gerez ML, López-Alquicira M, Cuellar-Ramírez A, et al. Epidemiología de las mordeduras por serpiente. Su simbolismo. Acta Pediatr Mex. 2009;30(3):182-91.

5. Secretaría de Salud. Boletín epidemiológico. Sistema Nacional de Vigilancia Epidemiológica. Sistema Único de Información. México: Dirección General de Epidemiología. 2018;52(35):1-62.

6. Neri-Castro E, Bérnard-Valle M, Alagón-Cano A. Reptiles venenosos de México. Revista Digital Universitaria [Internet]. 2014;15(11). Disponible en: http://www.revista.unam.mx/vol.15/num11/art86/art86.pdf
7. Siria-Hernández CG, Arellano-Bravo A. Mordeduras por serpiente venenosa: panorama epidemiológico en México. Salud Publica Mex. 2009;51(2):95-6.

8. Flores-Villela O, Goyenechea I. Patrones de distribución de anfibios y reptiles en México. En: Morrone JJ, Llorente Bousquets J, editores. Una perspectiva latinoamericana de la biogeografía. México: CONABIO, Facultad de Ciencias, Universidad Nacional Autónoma de México; 2003. pp. 289-296.

9. Grismer LL. The amphibians and reptiles of Baja California, its Pacific islands, and the islands in the Sea of Cortes: natural history, distribution and identification. Berkeley: University of California Press; 2002.

10. Uetz P, Freed P, Hošek J, editores. The Reptile Database [Internet]. The Reptile Database [última actualización: 22 de mayo de 2020; consultado: julio de 2020]. Disponible en: http://www.reptile-database.org

11. García E. Climas. Catálogo de metadatos geográficos. Comisión Nacional para el Conocimiento y Uso de la Biodiversidad; 1998.

12. Corral JAR, Medina García G, Meza Sánchez R, Padilla G, Serrano Altamirano V. Estadísticas climatológicas básicas del estado de Baja California (periodo 1961-2003). Libro Técnico Núm. 1. Ciudad Obregón, Sonora, México: INIFAP-CIRNO; 2006.

13. Salinas-Zavala CA, Leyva-Contreras A, LluchBelda D, Díaz-Rivera E. Distribución geográfica y variabilidad climática de los regímenes pluviométricos en Baja California Sur, México. Atmósfera. 1990;3:217-37.

14. González-Abraham CE, Garcillán PP, Ezcurra E. Ecorregiones de la Península de Baja California: Una síntesis. Bol Soc Bot Mex. 2010;87:69-82.

15. Arnaud G, Carbajal-Saucedo A, Pozas-Ocampo F. Comprendiendo el veneno de las serpientes de cascabel: componentes, efectos y uso potencial. Áreas Naturales Protegidas Scripta. 2019;5(2):39-56.

16. Zúñiga-Carrasco IR, Caro-Lozano J. Aspectos clínicos y epidemiológicos de la mordedura de serpientes en México. Evid Med Invest Salud. 2013;6(4):125-36

17. Instituto Nacional de Estadística y Geografía. Población rural y urbana. Censos y conteos de población y vivienda. México: Gobierno de México, Instituto Nacional de Estadística y Geografía; 2019.

18. Gobierno de Baja California. Apuntes de población de Baja California. México: COPLADE; 2013.

19. Gobierno de Baja California Sur. Demografía. Anuario Estadístico. México: Secretaría de Salud; 2017.

20. Martínez-Piedra R, Loyola-Elizondo E, Vidaurre-Arenas M, Nájera-Aguilar P. Paquetes de programas de mapeo y análisis espacial en epidemiología y salud pública. Bol Epidemiol OPS. 2004;25(4):1-9.

21. Leynaud GC, Reati GJ. Identificación de las zonas de riesgo ofídico en Córdoba, Argentina, mediante el programa SIGEpi. Rev Panam Salud Publica. 2009;26(1):64-9.

22. Arnaud G, Cordero-Tapia A, Ortíz-Avila V, Moctezuma-González CE, Tejocote-Pérez M, Carbajal-Saucedo A. Comparison of biological and biochemical characteristics of venom from rattlesnakes in the southern Baja California Peninsula. Toxicon. 2018;148 (2018):197-201.

23. Gil-Alarcón G, Sánchez-Villegas MC, Reynoso VH. Tratamiento prehospitalario del accidente ofídico: revisión, actualización y problemática actual. Gac Med Mex. 2011;147:195-208.

24. Almaraz-Vidal D. Las serpientes venenosas de importancia médica de la región de Las Grandes Montañas de Veracruz, México: aspectos ecológicos y accidentes ofídicos. Revista Mundo Investigación. 2016;2(1):173-80.

25. Juárez-Villa JD, López-de León J, Rodríguez-Mandujano M, Echegollen-Guzmán A. Mordedura de serpiente en el Hospital General de Ciudad Victoria, Tamaulipas. Evid Med Invest Salud. 2016;9:92-7.

26. Luna-Trejo J. Análisis epidemiológico del accidente por animales venenosos en el Estado de México del 2004 al 2016. Tesis de licenciatura en biología. Toluca, Estado de México: Universidad Autónoma del Estado de México, Facultad de Ciencias; 2018.

27. Matute-Martínez CF, Sánchez-Sierra LE, Barahona-López DM, Laínez-Mejía JL, Matute-Martínez FJ, Perdomo-Vaquero R. Caracterización de pacientes que sufrieron mordedura de serpiente, atendidos en hospital público de Juticalpa, Olancho. Rev Fac Cienc Med. 2016(enero-junio):18-26.

28. Rojas-Bárcenas AM. Informe de evento. Accidente ofídico [Internet] Colombia: Instituto Nacional de Salud; 2017. Disponible en: https://www. ins.gov.co/buscador-eventos/Informesdeevento/ACCIDENTE\%200F\%C3\%8DDICO_2018.pdf

29. Sevilla-Sánchez MJ, Mora-Obando D, Calderón JJ, Guerrero-Vargas JA Ayerbe-González S. Accidente ofídico en el departamento de Nariño, Colombia: análisis retrospectivo, 2008-2017. Biomédica. 2019;39:715-36.

30. Vélez-Alarcón LH, Real-Cotto JJ, Idrovo-Castro KJ, Alvarado-Franco HJ, Jaramillo-Feijoo LE, Ordóñez-Sánchez JL. Caracterización del accidente por mordedura de serpiente, atendidos en Unidades de Salud, Zona 5, Ecuador. Revista Científica INSPILIP. 2019;3(1). 